Fecha de recepción: agosto 2008 Fecha de aceptación: marzo 2009 Versión final: diciembre 2010

\section{La Educación Superior como un espacio de construcción del Patrimonio Cultural Una forma de entender la diversidad.}

Virginia Pineau ${ }^{*}$

\begin{abstract}
Resumen: La realidad de la educación superior en Argentina ha ido transformándose en los últimos años. Esto se debió, en parte, a lo que llamamos proceso de internalización de algunos de los grupos que la componen. Este proceso se refiere al incesante arribo a nuestro país de estudiantes y profesores de distintos países de América Latina.

El objetivo de este trabajo es analizar, desde una perspectiva antropológica, el rol que ocupa la educación superior en general y la educación universitaria en particular como un espacio de construcción del patrimonio cultural. A partir de entonces podremos analizar la diversidad cultural presente en las aulas universitarias en Argentina como consecuencia de los procesos migratorios más recientes.

Sin embargo, en este trabajo no entendemos al patrimonio como un concepto estático. Por tanto, haremos un análisis histórico del concepto para arribar a un nuevo concepto que se adapte a la realidad actual. Finalmente, veremos que es esta diversidad de la universidad Argentina la que favorece los procesos de construcción-reconstrucción, significación-resignificación y producción-reproducción del patrimonio cultural.
\end{abstract}

Palabras claves: diversidad cultural - patrimonio cultural - procesos de construcción-reconstrucción.

[Resúmenes en inglés y portugués y currículum en las páginas 94-95]

\title{
Introducción
}

La antropología es la ciencia que estudia al hombre y su cultura e intenta explicar y comprender la naturaleza de la diversidad cultural o de las diferencias culturales. Uno de los objetivos de la antropología es la desnaturalización de los conceptos. Esto es, a partir del recorrido histórico del concepto, comprenden su construcción como parte de la cultura y no como algo dado por la naturaleza.

Pero incluso el mismo concepto de cultura ha cambiado no sólo como consecuencia de la historia de la disciplina (que no llega a los 200 años) sino, por sobre todo, como resultado de la historia de la humanidad (Geertz 1987). Sin embargo, estas diferentes acepciones de cultura aún conviven en nuestra sociedad del siglo XXI.

La diferencia entre alta y baja cultura tiene sus comienzos hacia el siglo XVIII. La alta cultura es aquella cultura que merece ser conservada, la verdadera cultura. Se incluye en este grupo al arte pero no en una amplia acepción. Es el arte producido por y para cierto sector social. Es alta cultura (y por lo tanto verdadera y legítima cultura) la música clásica, la ópera, el ballet, las artes plásticas consagradas, cierta literatura clásica, entre otras.

A principios del siglo XX, el antropólogo norteamericano Franz Boas introdujo la idea de pluralidad cultural. No sólo era importante la "cultura" en singular, sino el estudio de "culturas" específicas. Una 
cultura particular sólo es comprensible a partir de su historia.

En la actualidad, el debate sobre el concepto de cultura en antropología no está cerrado pero sí se establecieron varios puntos de acuerdo. Por un lado, se considera que no existe una alta cultura y una baja cultura. La cultura es el conjunto de los conocimientos, creencias y hábitos que el ser humano adquiere como miembro de una sociedad. Por el otro lado, no existe una única cultura sino culturas que conviven y coexisten tanto entre los diferentes grupos humanos como al interior de los mismos. El concepto de patrimonio cultural no ha escapado al análisis antropológico (Prats 1997 y 1998). Como veremos a continuación, ha pasado de ser un conjunto de bienes que son preservados en lugares "sagrados" durante el siglo XIX a algo que está en constante construcción y que sólo puede ser "preservado" en parte, hacia fines del siglo XX. Con este trabajo nos proponemos arribar a una nueva y más completa definición del patrimonio cultural que se ajuste a la realidad del siglo XXI.

Las universidades juegan un rol fundamental en la construcción, legitimación y preservación del patrimonio cultural en este nuevo siglo. Por lo tanto, se analizará cómo las universidades llegaron a ocupar este rol y cómo el proceso de internacionalización de fines del siglo XX, promovió y desarrolló la construcción y creación de nuevo patrimonio cultural afectando tanto a los alumnos que asisten a las universidades así como a los docentes que en ellas enseñan e investigan.

\section{Una historia del término Patrimonio Cultural}

Con el fin de realizar una historia del concepto del patrimonio cultural seguiremos algunos de los conceptos desarrollados por Llorenc Prats (1998). En primer lugar, definiremos al patrimonio cultural como todo aquello que una sociedad considera merecedor de ser conservado más allá de que tenga o no un interés utilitario. En este concepto también incluimos a lo que se considera Patrimonio Natural como pueden ser los bosques o los seres vivos que los habitan ya que estos elementos naturales han sido seleccionados por los seres humanos.

El patrimonio cultural es tanto una invención como una construcción social. Es una invención porque implica la generación de discursos sobre la realidad con la intención de que éstos sean naturalizados. Es decir, que sean considerados como algo que no constituye una creación cultural sino algo dado por la naturaleza y por tanto inobjetable e inmutable. Y es una construcción social porque está asociada a los procesos de legitimación, esto es, de asimilación social de estos discursos más o menos inalterados. Entonces podemos afirmar que ninguna invención obtiene autoridad hasta que no es legitimada como construcción social. Aún más, ninguna construcción social se produce en forma espontánea sin haber previamente un discurso inventado por el poder (Foucault 1992)

Por lo tanto, el origen del patrimonio cultural se corresponde con un proceso de representación y legitimación simbólica de las ideologías. Es decir que consiste en la legitimación de referentes simbólicos a partir de fuentes de autoridad extraculturales, esenciales y, por lo tanto, inmutables. Estas fuentes convergen en los elementos culturales que están asociados con una identidad determinada y con ideas y valores. La identidad, las ideas y valores asociadas a los elementos culturales y el discurso que se genera adquieren, por lo tanto, un carácter sagrado y, entonces, esencial, indiscutible e inmutable. Todo este proceso surge, originariamente, con el Romanticismo. Este movimiento espiritual y artístico significó una reacción contra la razón y los demás cánones ilustrados. Una oposición del individuo hacia el Estado y del liberalismo contra el despotismo ilustrado.

Todos los elementos que pueden ser considerados patrimonializables pueden graficarse como incluidos en un triángulo. Los lados de este triángulo están compuestos por la naturaleza, la historia y la 
genialidad. Estos elementos son integrados en una dinámica de inclusión y exclusión considerablemente rígida. Es decir que, cualquier cosa, ya sea material o inmaterial, que proviene de la naturaleza, de la historia o de la genialidad son incluidos en los límites de este triángulo imaginario. Cualquier otro elemento que no provenga de ellos no será incluido. Por tanto, el contenido total de este triángulo conforma un conjuntos virtual de referentes simbólicos patrimoniales. Esto no significa que todos los elementos que integran el triángulo forman parte automáticamente del patrimonio sino que tienen la capacidad de serlo.

Sin embargo, este conjunto virtual no tiene una existencia real. No es tampoco la suma de todos los referentes patrimoniales activados por los museos y las instituciones sino que podría entenderse como un depósito inmenso y abstracto de posibilidades. Es decir, se trata de un repertorio hipotético de todos los referentes patrimoniales posibles. Los patrimonios que sí tienen una existencia real son aquellas colecciones activadas de los referentes patrimoniales que provienen del conjunto virtual. Podemos incluir, entre otros, a las estatuas, los monumentos, las colecciones de museos, los edificios, etc. Estas colecciones son activadas por las diferentes y diversas versiones ideológicas de la identidad.

La identidad es también una construcción social. Antes que una esencia, algo estático, inmutable y estrechamente limitado puede entenderse desde una posición relacional. Es decir, la identidad en relación y en oposición a otras identidades. Desde una perspectiva diacrónica, las identidades son cambiantes, fluyen y sus límites son imprecisos. Una identidad es una versión de esa identidad que puede coexistir con distintas versiones de una misma identidad que habitualmente se articulan en relaciones de complementariedad u oposición. Las representaciones patrimoniales afectan a los diferentes tipos de identidades pero lo hacen fundamentalmente con las identidades políticas que podemos llamar básicas. Estas son las identidades locales, regionales y nacionales.

Las identidades políticas no surgen a contramano de los movimientos culturales contemporáneos en los que están inmersos. Por lo tanto, debemos analizar al Romanticismo y el impulso que éste le dio a los nacionalismos, los pannacionalismos y los colonialismos como una forma de explicar el ascenso de la burguesía que necesitaba una política liberal que no impusiera trabas, límites o fronteras a los negocios. El Romanticismo y por lo tanto la burguesía, se sirvió, impulsó y construyó los nacionalismos para intentar convertir a la patria en intrínsecamente buena y a la empresa capitalista en un servicio a la patria y en una expresión de sus valores esenciales.

El colonialismo también fue impulsado por este movimiento aunque de forma indirecta. Se fomentó el espíritu de aventura y la épica de los exploradores. Aún más, se construyó un corpus científico para la validación del colonialismo con la creación de la antropología y de otras ciencias sociales que lo justificaban y lo exhibían como una labor humanitaria de auxilio a los países exóticos en su irremediable camino hacia la civilización.

Finalmente, los pannacionalismos forman parte de los dos modelos anteriores y se extienden dentro de la dinámica de la integración de pueblos, fuerzas productivas y mercados y de la lucha entre las potencias industriales europeas por la hegemonía. Para garantizar la eficacia de estas construcciones políticas las mismas deben ser formalizadas, explicadas, representadas y legitimadas ideológicamente (Anderson 2006). Aún más, es necesario que las mismas penetren intensamente en el tejido social. Es por esto que, todo el siglo XIX haya sido un período de una efervescencia identitaria. Para lograr esto se recurrió a todo tipo de doctrinas, sistemas de símbolos y representaciones como pueden ser las patrimoniales. El siglo XIX, por lo tanto, es el siglo de oro del patrimonio nacional en gran parte de Europa. Esto se manifiesta claramente en los museos que son el reservorio de un sentimiento nacional y patriótico. Las colecciones del British Museum, por ejemplo, ya no son consideradas como 
botines de guerra sino como una manifestación clara de la superioridad inglesa y de su inmensa obra de civilización y salvaguarda de la cultura. A lo largo de este trabajo veremos cómo esto se va modificando hasta el actual siglo XXI en donde entran en juego otras instituciones que custodian el patrimonio cultural como las escuelas o las universidades.

Pero ¿qué queremos decir cuando hablamos de activar el repertorio patrimonial? Significa elegir algunos referentes del conjunto y presentarlos de una manera o de otra. Como es evidente, esto significa articular un discurso que será acreditado por lo sagrado de los referentes. Este discurso obedecerá entonces de los referentes escogidos, de los significados de estos referentes que se destaquen, de la importancia relativa que se les otorgue, de su interrelación y del contexto. Por lo tanto, claramente, ninguna activación del repertorio patrimonial, ninguna de ellas, es neutral o inocente, ya sea de forma inconsciente o consciente por parte de los gestores del patrimonio cultural.

Aún más, ¿quién o quiénes activan estas diferentes versiones del conjunto del repertorio patrimonial? Claramente no es la sociedad ni ningún sujeto colectivo. Por el contrario, el rol de la sociedad es el de adherir y/o otorgar u oponerse y/o denegar, consensuar una representación, una imagen, un discurso y siempre en grado y forma variable según los distintos individuos. Esta representación, este discurso, esta imagen fueron elaborados por individuos concretos y reales al servicio consciente o inconsciente de ideas, valores e intereses concretos. La imagen del sujeto colectivo o de la sociedad es utilizada para naturalizar estos procesos.

La activación del repertorio patrimonial es tarea, en primer lugar, de los poderes constituidos: el poder político principalmente (gobiernos locales, regionales o nacionales). Esto no implica que otros poderes (como el poder económico, por ejemplo) no tengan la posibilidad o la capacidad de activar repertorios patrimoniales sino que, en líneas generales, están difícilmente interesados en proponer versiones de una determinada identidad.

Sin embargo, no solamente el poder político legalmente constituido, como los diferentes niveles de gobierno, pueden construir patrimonios. Existe un poder político informal y/o alternativo que también construye patrimonios. Más aún cuando se trata de poderes políticos informales opuestos al poder político constituido ya que la confrontación que se establece no tiene lugar en la arena política del estado ni en sus instituciones. Por lo tanto, resulta enorme el valor de las versiones alternativas de la identidad representadas por los repertorios patrimoniales aunque estas no se puedan encontrar en los museos. Esto no significa que no estén presentes ni que no sean conocidos por la colectividad ideológica.

Finalmente, los repertorios patrimoniales también pueden ser activados desde la sociedad civil por diferentes agentes sociales. En este caso, deben contar con el sustento o, al menos la condescendencia, del poder. En síntesis, sin poder no existe el patrimonio.

Las distintas versiones de la identidad que están representadas en los repertorios patrimoniales activados constituyen entonces la expresión de los diferentes "nosotros" del "nosotros". Su validez relativa puede medirse por la cantidad y por la calidad de las aprobaciones resultantes. Estas aprobaciones, a su vez, legitiman sistemas, políticas, estado de cosas y acciones concretas.

Estos referentes patrimoniales, al ser considerados sagrados, necesitan de un entorno adecuado que asegure su conservación y contemplación. Por lo tanto, los museos se transformaron en el siglo XIX en los templos que custodiaron estos referentes y, junto con esto, de las ideas, de los valores y, por tanto, de la identidad que expresan. Más adelante veremos cómo el rol de los museos se fue transformando con el correr de los siglos y cómo surgieron nuevos lugares o espacios que se transformaron en custodios de los referentes patrimoniales.

Al finalizar la Segunda Guerra Mundial se produjeron algunas transformaciones que afectarían irre- 
vocablemente algunos hábitos de la sociedad occidental con respecto al uso del tiempo libre y del ocio. Entre otros es significativo mencionar el desarrollo de lo que se llamó la revolución de los transportes (ya presente en el siglo XIX pero con fines mercantiles), la amplia generalización de las vacaciones pagas y con esto una mayor disponibilidad de recursos por parte de la clase media. En la década del sesenta, y como consecuencia de estas condiciones, se advierte que se está constituyendo un mercado floreciente. Se desarrolla entonces el turismo a escala planetaria. Junto con esto, el otro gran factor que va a transformar extremadamente los hábitos, sobretodo, de la clase media es la revolución de las telecomunicaciones, principalmente la televisión. Esto no solamente va a cambiar los hábitos sino también nuestra propia percepción de la realidad. Desde entonces, la realidad desde la vida cotidiana más cercana hasta los grandes procesos históricos mundiales, como las guerras o la caída del Muro de Berlín por ejemplo, están presentes en tiempo real en los livings de las casas. Por tanto, esta realidad se desnaturaliza y adquiere un carácter virtual.

Para la segunda mitad del siglo XX, entonces, el turismo y la televisión nos permiten vivir otras realidades además de la propia vida cotidiana. Esto nos ha habituado también a convertir a la realidad en un espectáculo y a nosotros en espectadores que contemplan esa realidad aunque eso implique miserias y guerras. Aún más, la economía de mercado nos ha acostumbrado a que todo se pueda convertir en artículo de consumo, aunque sea como un espectáculo de la miseria y de las guerras, y que, por tanto, se pueda adquirir con dinero.

Veamos ahora cómo estos nuevos elementos afectaron al ámbito del patrimonio. A partir de este momento, no solamente los cuadros y los monumentos son espectáculos y por tanto artículos de consumo. Fiestas, tradiciones, procesos productivos (como la ruta del vino) y culturas pueden ahora observarse (y consumirse) desde el televisor o en vivo a través del turismo. Esto no significa que la relación entre patrimonio y turismo sea algo novedoso. Al contrario, el patrimonio ha sido uno de los primeros motivos de los viajes aún antes del turismo como lo conocemos hoy. La diferencia es que con la espectacularización de la realidad y con la masificación del turismo se produce un cambio cuantitativo y cualitativo en la asociación entre patrimonio y turismo. Por un lado, los destinos patrimoniales clásicos se ven sometidos a una presión turística muy intensa que puede poner en peligro su conservación. Sucesivamente, los destinos patrimoniales previamente activados entran en la lógica del espectáculo y del consumo y se adaptan a las nuevas demandas y necesidades expositivas para no quedar marginados del mercado. Finalmente, se activan aquellos repertorios patrimoniales que hasta entonces eran inviables por su accesibilidad gracias a la mejora en los desplazamientos y a la creciente demanda de atracciones turísticas.

Los museos, las instituciones más clásicas del patrimonio, no son ajenas a estas transformaciones. Las activaciones de repertorios patrimoniales, viejas y nuevas, se miden ahora fundamentalmente no por la cantidad o la calidad de adhesiones sino por el consumo (en este caso el número de visitantes). Se incrementan las exposiciones temporales lo que significa una renovación de la oferta. Además se incorporan, incluso de forma frenética, innovaciones en las técnicas expositivas a las que se les incorpora en forma inmediata cualquier novedad tecnológica. Todo esto implica el surgimiento de nuevos tipos de activaciones patrimoniales cuya motivación no es ya de carácter identitario sino abiertamente turística y comercial. Desde entonces, los referentes se activan y los significados que se les confieren no responden a los diversos "nosotros del nosotros" que representan las diversas versiones ideológicas de la identidad sino, principalmente, al "nosotros de los otros". Esto es, a la imagen externa y comúnmente estereotipada que se tiene de nuestra identidad desde los centros emisores del turismo. Si tomamos como ejemplo la imagen del argentino que se vende al exterior incluiríamos 
sin dudar al mate, el asado y el tango. Por supuesto que esta lista no sólo es poco representativa del total del país (sólo se baila tango en Buenos Aires) sino que además es un estereotipo y no representa al argentino real.

Estamos entonces ante otras activaciones del patrimonio. Y por lo tanto, de representaciones de la identidad. Sin embargo, en este caso estas activaciones pueden provocar confrontaciones entre la lógica turístico-comercial y la lógica identitaria. Frecuentemente, estas activaciones han nacido como consecuencia de la masificación del turismo. Las poblaciones locales pueden ver peligrar su identidad como consecuencia de este fenómeno. Sin embargo, esto no es un impedimento para que se adapten a estas imágenes externas de esta misma identidad. Por una parte, porque las poblaciones locales no podrían evitar el fracaso al carecer de visitantes. Por otra parte, porque esta misma imagen al ser reproducida por los discursos hegemónicos a través de los medios masivos de comunicación y, aún más, del sistema educativo, ha sido adoptada por la propia población local como la visión de sí mismos, como la denominada "memoria colectiva".

¿Qué pasa entonces con los museos ante esta nueva situación? Si excluimos a todos aquellos que por sí mismos son casos excepcionales, como los grandes museos europeos y norteamericanos, los museos se convirtieron en instituciones obsoletas que ya no atraen visitantes y, por lo tanto, discordantes con la nueva lógica de mercado de estos tiempos. Se les exige ahora un cambio en su forma de presentar las colecciones. Se generalizan las exposiciones transitorias y la continua renovación de las técnicas expositivas.

Con respecto a la relación museo-sociedad, se observan dos direcciones distintas. Por un lado se intenta hacer que las colecciones sean más accesibles y comprensibles a una mayor cantidad de visitantes. Nacen entonces los museos de la ciencia o los museos interactivos. Por el otro lado, se hace más sensible a la demanda social, desde las modas más superficiales hasta sus preocupaciones más acuciantes.

\section{Hacia un nuevo concepto de Patrimonio Cultural}

La especie humana tiene un único patrimonio biológico y un único patrimonio cultural que no se transmite genéticamente sino mediante el aprendizaje. Entonces existe un solo patrimonio cultural humano constituido por todo aquello creado por la especie. Como ejemplo de este patrimonio podemos mencionar cosas tan diversas como un sistema de creencias o de rituales, el arte, el conocimiento científico, el conocimeinto técnico, etc. Debemos incluir también entre estos ejemplos a los errores que la humanidad cometió a lo largo de su historia.

Sin embargo, existe una diferencia fundamental entre ambos patrimonios. El patrimonio biológico se puede conservar y se transmite genéticamente. Por el contrario, el patrimonio cultural se transmite por imitación, aprendizaje y herencia. En el proceso de uso de este patrimonio cultural el mismo se transforma, se innova, algunos elementos dejan de usarse o se resignifican o adquieren nuevas funciones. La cultura, entendida como todo aquello que es creación humana desde un sistema de parentesco hasta una innovación tecnológica, cambia y se transforma inevitablemente a lo largo del tiempo. Aún más, este cambio se acentúa porque no se trata de un proceso de reproducción mecánica sino de transmisión cultural.

¿Cómo hacemos entonces para conservar la cultura? Lamentablemente esto no es posible. Lo que sí puede ser conservado es el conocimiento que genera esa cultura. De esto se han ocupado las ciencias sociales en general y la antropología en particular. Este es el verdadero patrimonio cultural que la humanidad puede conservar y, por lo tanto, transmitir. Nos referimos no sólo al conocimiento 
científico sino también al arte, los sistemas de parentesco, la tecnología y todo aquello que le permitió al hombre adaptarse a entornos tan cambiantes en términos geográficos y también en procesos sociohistóricos.

Como es evidente, es imposible conservar el conocimiento absoluto de las culturas debido a su extremada complejidad. Por otra parte, no tendría sentido intentar conservar este conocimiento de una forma puramente descriptiva. Las culturas son sistemas que cambian. No es posible abarcarlas en su totalidad, ni congelarlas en el tiempo ni reducirlas a un conjunto innumerable de datos inconexos cuya suma no ofrece ningún resultado. En suma, no podemos conservar la cultura, ni el conocimiento de la cultura sino sólo parte de este conocimiento. Sin embargo, esta parte que se conserva también estará determinada por criterios e intereses utilitarios y actuales. Es este, entonces, el patrimonio cultural que podemos aspirar a conservar, comunicar y transmitir.

El medio más adecuado para la formalización de este patrimonio cultural parecería ser la ciencia. Sin embargo, ni la ciencia ni la razón son las únicas formas posibles de conservar el conocimiento de la diversidad cultural. El arte es un ejemplo de esto. Se trata de la expresión del conocimiento artístico que no se formaliza más que en él. Es decir, es inseparable de sus referentes, al margen y más allá de su manipulación ideológica y de sus connotaciones simbólicas. Existe en el arte una aprehensión del mundo que no permite reducirlos a meros epifenómenos culturales científicamente interpretables. En definitiva, para la antropología, la definición de patrimonio cultural coincide con esta concepción del patrimonio cultural como conocimiento (incluyendo el conocimiento artístico) más que con la concepción habitual del patrimonio cultural como un conjunto de bienes o reliquias como fuimos detallando a lo largo de este trabajo. Sin embargo, esto no es un impedimento para que, a pesar de nuestras certezas, las activaciones patrimoniales que sigan funcionando socialmente continúen siendo las de siempre: las activaciones del patrimonio al servicio de la identidad para nosotros y las activaciones para los otros, es decir, para vender en el mercado turístico.

\section{La Universidad como un ámbito de producción del Patrimonio Cultural}

La historia de la educación universitaria en Argentina es incluso más antigua que el propio país. En 1610 la Compañía de Jesús creó el Collegium Maximum, en la ciudad de Córdoba, donde asistían alumnos religiosos de esa orden. Ésta fue la base de la universidad, que era en esa época de la más alta categoría intelectual. Cuando estaba en manos de los jesuitas y regido bajo el Obispo Juan Fernando de Trejo y Sanabria, en 1613, se iniciaron los Estudios Superiores en el Colegio Máximo de Córdoba, aunque no contaba aún con la autorización para conceder grados. Esto se lograría recién el 8 de agosto de 1621.

Desde entonces, el rol de la universidad en la Argentina y en Latinoamérica fue cambiando y adaptándose a las nuevas realidades tanto nacional como internacional. Lértora Mendoza (2002) plantea la existencia de tres etapas en la historia de las universidades latinoamericanas. Una primera etapa que la llama colonial (siglos XVII y XVIII), con sus sub-etapas escolástica, ecléctica e ilustrada. Una segunda etapa llamada independiente (principios del XIX), con sus sub-etapas revolucionaria, organizativa y positivista y reforma y modernización (c.1910-1930) con los caracteres comunes de profesionalización, democratización, desarrollismo, normalización de la ciencia nacional, transferencia de tecnología y, socialización del conocimiento. Finalmente, una época actual, caracterizada por el proceso de globalización.

La revolución de las comunicaciones y de los medios de transporte después de la Segunda Guerra 
Mundial no sólo favoreció la generación de patrimonio cultural a través de la masividad del turismo como vimos anteriormente. Como sostiene Peter Burke (2001) los asuntos, las necesidades y las temáticas de interés y preocupación de nuestra época parecen ayudar a pensar, re-pensar e interpretar el pasado humano. Justamente es en el marco general de la denominada globalización donde se construyen y constituyen las identidades referentes de diversos grupos humanos de distintas regiones y la constante renovación tecnológica, así como el consumo de la misma, juega un rol importante en dichos procesos.

El proceso de globalización originado hacia fines del siglo XX permitió el desvanecimiento de las fronteras y un intercambio de información constante e ininterrumpido. Las universidades argentinas se vieron afectadas por este proceso en dos de sus pilares fundamentales: los docentes y los alumnos. Con respecto a los docentes de las universidades argentinas, la revolución de las comunicaciones les permitió una entrada más rápida y eficaz a los centros generadores de conocimiento en el extranjero. Esto se observa en un doble flujo: tanto para acceder a la información producida en las universidades del exterior (como bibliotecas virtuales, libros, congresos, etc.) así como para poder hacer pública la información que producen los docentes en nuestro país. Aún más, fue posible el encuentro con docentes del extranjero a partir de la organización, en y por parte de, las universidades argentinas de conferencias, talleres, bienales, entre otros ejemplos.

Los alumnos de las universidades argentinas se vieron favorecidos, en primer lugar, por los mismos beneficios que los docentes debido a que son ellos quienes los forman en las universidades. Por otra parte, la revolución en los transportes permitió y fomentó lo que llamamos proceso de internacionalización. Este proceso se desarrolló y se desarrolla entre la última década del siglo XX y la primera década del siglo XXI. Durante estos años, miles de personas migraron de países limítrofes y de otros países latinoamericanos ya sea para asentarse en la Argentina como para estudiar en sus universidades. Este proceso de internacionalización tuvo como consecuencia un aumento de la diversidad cultural presente en las aulas argentinas. Esto se refleja en dos resultados que, si bien parecieran opuestos a primera vista, son claros reflejos de la globalización. Por un lado, un aumento de los regionalismos, esto es, las características propias de cada grupo o cultura de los alumnos. Por el otro, la mixtura o la amalgama de estos regionalismos favorece la creación o surgimiento de mayor diversidad cultural.

Entonces, esta diversidad cultural, estos elementos diferentes que se ponen en juego en las aulas, promueven la construcción y la reconstrucción del patrimonio cultural existente dando como resultado un nuevo patrimonio. Esta es una de las formas en las que las universidades construyen y crean patrimonio cultural.

Otra forma de construir y reconstruir el patrimonio cultural en las universidades se relaciona con el concepto antropológico del patrimonio cultural. Al entender al patrimonio cultural como el conocimiento que genera una cultura, las universidades ocupan un lugar central en este proceso. Son el lugar en donde el conocimiento se crea, se transmite, se conserva y se analiza.

En conclusión, como consecuencia de la revolución de los transportes y de las comunicaciones ocurrida desde la segunda mitad del siglo XX, las universidades argentinas se transformaron en centros generados de diversidad cultural y, por tanto, de patrimonio cultural. Por otra parte, también les cabe a las universidades el rol de analizar y conservar el patrimonio cultural concebido como el conocimiento generado por una cultura en particular.

Los museos, con sus múltiples transformaciones ya no son las únicas instituciones encargadas de velar por el patrimonio cultural. Las universidades argentinas se renuevan y adquieren, en el siglo 
XXI, el lugar de custodios del patrimonio cultural. Son uno de los reservorios del conocimiento que construye cada cultura. Aun más, ellas mismas también generan nuevos conocimientos.

\section{Conclusiones}

$\mathrm{Al}$ inicio de este trabajo nos propusimos explicar y analizar el rol que ocupan las universidades como un espacio de construcción del patrimonio cultural desde una perspectiva antropológica.

Para cumplir con este propósito analizamos, en primer lugar, la historia del concepto patrimonio cultural con el fin de desnaturalizarlo y poder arribar a un concepto más adaptado al siglo XXI.

El siglo XIX fue el siglo de construcción y legitimación de las identidades nacionales en buena parte de Europa como consecuencia del colonialismo y como una manera de entender el ascenso de la burguesía. Esta nueva clase social necesitaba la integración de los pueblos, de las fuerzas productivas y de los mercados para sacar ventajas en la lucha por la hegemonía entre las potencias europeas. Esta integración debe estar garantizada a partir de la formalización, representación y la legitimación ideológica. Además, estas construcciones políticas deben adentrarse en el tejido social. Por ello, se uso a la identidad para lograr esta unión y, por lo tanto, a todo sistema de símbolos y representaciones como el patrimonio cultural. Los museos y sus colecciones se tornaron entonces en el lugar ideal para preservarlo.

En la segunda mitad del siglo XX y luego de finalizada la Segunda Guerra Mundial, se produjeron grandes transformaciones que influirían notablemente en el uso del tiempo libre de gran parte de la sociedad occidental: la revolución de los transportes, las vacaciones pagas y la revolución de las telecomunicaciones. Estas transformaciones crearon un mercado próspero: el turismo a escala mundial. A partir de entonces, no sólo los cuadros o los monumentos son artículos de consumo. Desde la pantalla del televisor o en vivo y en directo a través del turismo pueden ahora verse fiestas populares, tradiciones, procesos productivos, etc.

Sin embargo, el turismo y el patrimonio estuvieron relacionados desde incluso antes que este momento. Lo que hace único y distinto a esta relación es la masificación y el carácter espectacular del turismo. Esto traerá como consecuencia que los destinos clásicos del turismo estén sometidos a una muy fuerte presión turística y que se activen nuevos destinos gracias a las mejoras en los transportes y las comunicaciones y a la necesidad de nuevas atracciones turísticas.

Los museos también se ven modificados por estas transformaciones. El éxito de los repertorios patrimoniales se mide ahora en número de visitantes. Por tanto, las exposiciones temporales aumentan incluyendo además innovaciones tecnológicas en las exposiciones. En síntesis, existen ahora nuevos tipos de activaciones de los referentes patrimoniales. Ya no se trata de la identidad sino del éxito comercial. Finalmente, desarrollamos el concepto de patrimonio cultural desde el punto de vista antropológico. La antropología no ve al patrimonio cultural como a un conjunto de bienes o reliquias que se conservan en un museo. Por el contrario, ve al patrimonio cultural como al conocimiento de la cultura. Este conocimiento no se refiere sólo al conocimiento científico o al que se adquiere a partir de la razón sino también al conocimiento artístico. Todo este conocimiento está en constante construcción y debido a su complejidad sólo puede ser conservado en parte.

Otra de las consecuencias de la revolución de las comunicaciones y de la revolución de los transportes fue su impacto en las universidades argentinas. Estos centros de estudios se vieron favorecidos por lo que llamamos proceso de internacionalización. Todos estos procesos afectaron tanto a los alumnos como a los docentes. Por un lado, implicó la inclusión de nuevos alumnos provenientes de 
países latinoamericanos. Por el otro, para los docentes significó un acceso al conocimiento producido en otras partes del planeta a partir del uso de bibliotecas virtuales, entre otros recursos, así como la posibilidad de intercambiar conocimiento con otros profesionales a partir de la organización de talleres, congresos, muestras, etc.

Las universidades argentinas, por lo tanto, se constituyeron en centros generadores de diversidad cultural y, como consecuencia, de patrimonio cultural. Entonces, las universidades se constituyen, en el siglo XXI, como las encargadas de analizar y conservar el conocimiento generado por ellas mismas y por el resto de la comunidad. Esto es, el conocimiento que forma al patrimonio cultural.

\title{
Referencias Bibliográficas
}

Anderson, Benedict. (2006). Comunidades imaginadas. Reflexiones sobre el origen la difusión del nacionalismo. México: Fondo de Cultura Económica.

Ballart, Josep. (2002). El patrimonio histórico y arqueológico: valor y uso. Barcelona: Ariel.

Burke, Peter. (2005). “El renacimiento italiano y el desafió de la posmodernidad”. En: Schröder y Breuniger (comps.)

Teoría de la cultura. Un mapa de la cuestión. Buenos Aires: Fondo de Cultura Económica.

Comisión para la Preservación Histórico-Cultural de la Ciudad de Buenos Aires. Temas de Patrimonio Cultural II. Buenos Aires: Eudeba.

Endere, María Luz. (1995). “La arqueología de rescate en Argentina. Implicaciones legales y científicas”. Tesis para optar al título de Licenciada en antropología con orientación arqueológica. Facultad de Ciencias Sociales de Olavarría. Universidad Nacional del Centro de la Provincia de Buenos Aires. Ms.

Foucault, Michel. (1992). Microfísica del poder. Madrid: La Piqueta.

Geertz, Clifford. (1987). La interpretación de las culturas. Buenos Aires: Gedisa.

Lértora Mendoza, Celina. (2002). “Lineamientos para una historia de la universidad latinoamericana”. En: CUYO. Anuario de Filosofía Argentina y Americana, pp. 18-19.

Prats, Llorenc. (1998). “El concepto de patrimonio cultural”. En: Política y Sociedad. 27. (1997). Antropología y patrimonio. Barcelona: Ariel.

Rotman, Mónica. (ed) (2004). Antropología de la Cultura y el Patrimonio. Diversidad y desigualdad en los procesos culturales contemporáneos. Córdoba: Ferreyra Editor.

Wright, Pablo. (2003). “Símbolos y cultura: Sobre la materialidad del patrimonio intangible”. Temas de patrimonio. 7.

\begin{abstract}
Summary: The reality of superior education in Argentina has been transformed in the last years due to which we called process of insight of some of the groups involved in it. This process shows the ongoing arrival to our country of students and professors of different countries from Latin America. This work aims to analyze, from an anthropological perspective, the role occupied both by superior education and the university education in particular like a space of construction of the cultural patrimony. Starting from those premises, we will be able to analyze the cultural diversity in Argentina's University as a result of the more recent migratory processes. Otherwise, in this work we do not sustain patrimony as a static concept. Therefore, we will make an historical analysis of the concept to arrive at a new concept that adapts to the present reality. Finally, we will see that this diversity of the Argentina university allows the processes of construction-reconstruction, resignification and production-reproduction of the cultural patrimony.
\end{abstract}

Key words: cultural diversity - cultural patrimony - processes of construction-reconstruction. 
Resumo: A realidade da educação superior em Argentina foi transformando-se nos últimos anos. Isto se deveu, em parte, ao que chamamos processo de internalização de alguns dos grupos que a compõem. Este processo refere-se ao incessante aporto a nosso país de estudantes e professores de diferentes países de América Latina. O objetivo deste trabalho é analisar, desde uma perspectiva antropológica, o papel que ocupa a educação superior em geral e a educação universitária em particular como um espaço de construção do patrimônio cultural. Assim, poderemos analisar a diversidade cultural presente nas salas de aula universitárias em Argentina como conseqüência dos processos migratórios mais recentes. No entanto, neste trabalho nao entendemos ao patrimônio como um conceito estático. Por tanto, faremos uma análise histórica do conceito para aportar um novo conceito que se adapte à realidade atual. Finalmente, veremos que é esta diversidade da universidade argentina a que favorece os processos de construção-reconstrução, significação-resignificação e produçãoreprodução do patrimônio cultural.

Palavras chave: diversidade cultural - patrimônio cultural - processos de construção- reconstrução.

${ }^{(*)}$ Doctora en Arquelología (UBA). Licenciada en Ciencias Antropológicas, orientación Arqueología (U.B.A). Profesora de antropología para el nivel medio y terciario. Docente en nivel primario, medio y universitario. 